\title{
PeV Cosmic Ray Acceleration in the Supernova Post Breakout Expansion Phase: Kinetic-magnetohydrodynamic Simulations
}

\section{Tsuyoshi Inoue, ${ }^{a, *}$ Alexandre Marcowith, ${ }^{b}$ Gwenael Giacinti, ${ }^{c}$ Allard Jan van Marle ${ }^{b}$ and Shogo Nishino ${ }^{a}$}

\author{
${ }^{a}$ Department of Physics, Graduate School of Science, Nagoya University, \\ Furo-cho, Chikusa-ku, Nagoya 464-8602, Japan \\ ${ }^{b}$ Laboratoire Universe et Particules de Montpellier (LUPM) Université Montpellier, \\ CNRS/IN2P3, CC72, place Eugène Bataillon, 34095, Montpellier Cedex 5, France \\ ${ }^{c}$ Max-Planck-Institut für Kernphysik, \\ Saupfercheckweg 1, 69117 Heidelberg, Germany \\ E-mail: tsuyoshi.inoue@nagoya-u.jp
}

\begin{abstract}
Origin of cosmic rays (CRs) is still not known. In this work we argue that PeV CRs can be accelerated during the early phase of a supernova blast wave expansion in dense red supergiant winds. We solve in spherical geometry a system combining a diffusive-convection equation which treats CR dynamics coupled to magnetohydrodynamics to follow gas dynamics. The fast shock expanding in a dense ionized wind is able to trigger the fast non-resonant streaming instability over day timescales. We investigate the maximum energy CRs can reach in this configuration accounting for $\mathrm{pp}$ losses. Multi-PeV energies can be reached if the progenitor mass loss rates are of the order of, or larger than, $10^{-3} M_{\text {sun }} \mathrm{yr}^{-1}$. It has been recently invoked that prior to the explosion hydrogen rich massive stars can produce enhanced mass loss rates. These enhanced rates would then favor the production of a Pevatron phase in early times after the shock breakout. We discuss observational tests to probe our model using future radio and gamma-ray facilities.
\end{abstract}

$37^{\text {th }}$ International Cosmic Ray Conference (ICRC 2021)

July 12th - 23rd, 2021

Online - Berlin, Germany

\footnotetext{
*Presenter
} 


\section{Introduction and Basics}

Origin of galactic CRs, in particular that of PeVatron, is still highly unknown. Recent theoretical studies suggest that very early phase of supernova blast wave shock that expands in dense circumstellar medium (CSM) is a suitable site of cosmic-ray acceleration up to $1 \mathrm{PeV}$ or higher. In this study, we numerically examine particle acceleration in such circumstances by solving kineticmagnetohydrodynamics equations in spherical polar geometry [1, 2]:

$$
\begin{aligned}
& \frac{\partial \rho}{\partial t}+\frac{1}{r^{2}} \frac{\partial}{\partial r} r^{2}\left(\rho v_{r}\right)=0, \\
& \frac{\partial}{\partial t}\left(\rho v_{r}\right)+\frac{1}{r^{2}} \frac{\partial}{\partial r} r^{2}\left(\rho v_{r}^{2}+p+\frac{B_{\theta}^{2}+B_{\phi}^{2}}{8 \pi}\right)=0 \\
& \frac{\partial}{\partial t}\left(\rho v_{\theta}\right)+\frac{1}{r^{2}} \frac{\partial}{\partial r} r^{2}\left(\rho v_{r} v_{\theta}-\frac{B_{r} B_{\theta}}{4 \pi}\right)=-\frac{1}{c} j_{r}^{(\mathrm{ret})} B_{\phi}, \\
& \frac{\partial}{\partial t}\left(\rho v_{\phi}\right)+\frac{1}{r^{2}} \frac{\partial}{\partial r} r^{2}\left(\rho v_{r} v_{\phi}-\frac{B_{r} B_{\phi}}{4 \pi}\right)=\frac{1}{c} j_{r}^{(\mathrm{ret})} B_{\theta}, \\
& \frac{\partial \epsilon}{\partial t}+\frac{1}{r^{2}} \frac{\partial}{\partial r} r^{2}\left\{v_{r}\left(\epsilon+p+\frac{B^{2}}{8 \pi}\right)-B_{r} \frac{\vec{B} \cdot \vec{v}}{4 \pi}\right\}=-\frac{j_{r}^{(\mathrm{ret})}}{c}(\vec{v} \times \vec{B})_{r}, \\
& \epsilon=\frac{p}{\gamma-1}+\frac{1}{2} \rho v^{2}+\frac{B^{2}}{8 \pi}, \\
& \frac{\partial B_{\theta}}{\partial t}=\frac{1}{r^{2}} \frac{\partial}{\partial r} r^{2}\left(B_{r} v_{\theta}-B_{\theta} v_{r}\right), \\
& \frac{\partial B_{\phi}}{\partial t}=\frac{1}{r^{2}} \frac{\partial}{\partial r} r^{2}\left(B_{r} v_{\phi}-B_{\phi} v_{r}\right), \\
& \frac{\partial F_{0}(r, p)}{\partial t}+\frac{1}{r^{2}} \frac{\partial}{\partial r}\left\{r^{2} v_{r} F_{0}(r, p)\right\}-\frac{1}{3} \frac{\partial v_{r}}{\partial r} \frac{\partial F_{0}(r, p)}{\partial \ln p} \\
& \quad=-\frac{c}{3} \frac{1}{r} \frac{\partial}{\partial r}\left\{r F_{1}(r, p)\right\}+Q_{\mathrm{inj}}(r, p) p^{3}-L_{\mathrm{pp}}(r, p) p^{3}, \\
& \frac{\partial F_{1}(r, p)}{\partial t}+\frac{1}{r^{2}} \frac{\partial}{\partial r}\left\{r^{2} v_{r} F_{1}(r, p)\right\} \\
& \quad=-\frac{c}{r} \frac{\partial}{\partial r}\left\{r F_{0}(r, p)\right\}-\frac{c^{2}}{3 \kappa(p, \vec{B})} F_{1}(r, p)-L_{\mathrm{pp}}(r, p) p^{3},
\end{aligned}
$$

where $f_{0} \equiv F_{0} / p^{3}$ and $f_{1} \equiv F_{1} / p^{3}$ are respectively the isotropic and first order anisotropic $j_{r}^{(\text {ret })}$ is the return current density induced by the cosmic ray streaming current, $Q_{\text {inj }}$ is an injection rate that supply low-energy CRs at shock front with fraction $\eta=6 \times 10^{-4}$ of thermalized particles, $L_{\mathrm{pp}}$ is a momentum loss rate due here to inelastic p-p collisions, and $\kappa$ is the diffusion coefficient. We use the diffusion coefficient as follows [3, 4]

$$
\kappa(p, \vec{B})=\frac{4}{3 \pi} \frac{\max \left(B_{r}^{2}, \delta B^{2}\right)}{\delta B^{2}} \frac{v_{\mathrm{CR}} p c}{e \max \left(\left|B_{r}\right|, \delta B\right)},
$$

where $\delta B^{2}=B_{\theta}^{2}+B_{\phi}^{2}$, and $v_{\mathrm{CR}}$ is the cosmic-ray velocity at momentum $p$.

To mimic a CSM created by a red supergiant (RSG), we employ a model developed by [5], where we introduce a parameter $\varpi$ that describes conversion efficiency of RSG wind kinetic energy to CSM magnetic field by turbulent dynamo. Figure 1 shows a radial structure of the CSM density and magnetic field strength with mass loss rate of RSG wind $\dot{M}=10^{-5} M_{\text {sun }} \mathrm{yr}^{-1}$ and $\varpi=1$. 


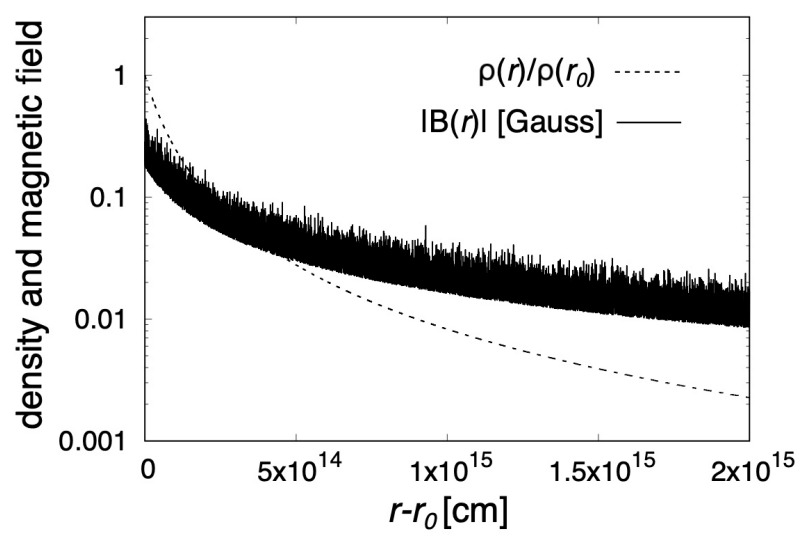

Figure 1: Initial CSM structure that is created by RSG wind of $\dot{M}=10^{-5} M_{\text {sun }} \mathrm{yr}^{-1}$. Dashed line shows the density normalized at the inner boundary $r_{0}=10^{14} \mathrm{~cm}\left(\rho_{0}=5 \times 10^{-15} \mathrm{~g} \mathrm{~cm}^{-3}\right)$. Solid line shows the initial magnetic field strength $|B(r)|=\left\{B_{r}(r)^{2}+\delta B(r)^{2}\right\}^{1 / 2}$ under $\varpi=1$.

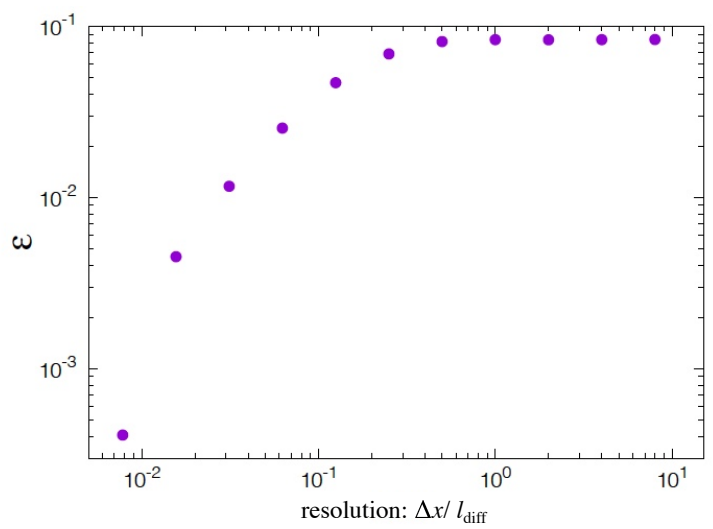

Figure 2: Result of test simulations of DSA. $\epsilon$ is the deviation of power-law index of CR distribution function at shock front from the DSA theory.

\section{Performance of our code}

We solve the above equations using the code developed by [2]. To exhibit the reliability of the code, we show results of two kinds of basic test simulations. First test shows reproducibility of the diffusive shock acceleration (DSA) spectrum by [6], which predicts $f_{0}\left(x_{\mathrm{sh}}, p\right) \propto p^{-4}$ for high Mach number shock wave. Figure 2 is the plot of deviation $\epsilon$ of the numerical spectral index of $f_{0}\left(x_{\mathrm{sh}}\right)$ from the analytic solution as a function of spatial resolution of diffusion length $l_{\text {diff }} / \Delta x=\kappa / v_{\text {sh }} / \Delta x$. We can see that our code reproduces analytic solution of the DSA very well $(\epsilon \lesssim 0.1)$ even if the diffusion length is marginally resolved. Since the non-resonant CR streaming instability ([7]; here after the Bell instability) is able to grow in our system, we also test numerical growth rate of the Bell instability. In Figure 3, we compare the numerical dispersion relation with the analytic one under constant CR current, i.e., $\sigma(k)^{2}=v_{\mathrm{a}}^{2} k^{2}-B_{0} j^{\text {(ret) }} k /(\rho c)$. From left panel to right panel, numerical spatial resolution against the most unstable scale is increased from 16 to 64 spatial cells. 

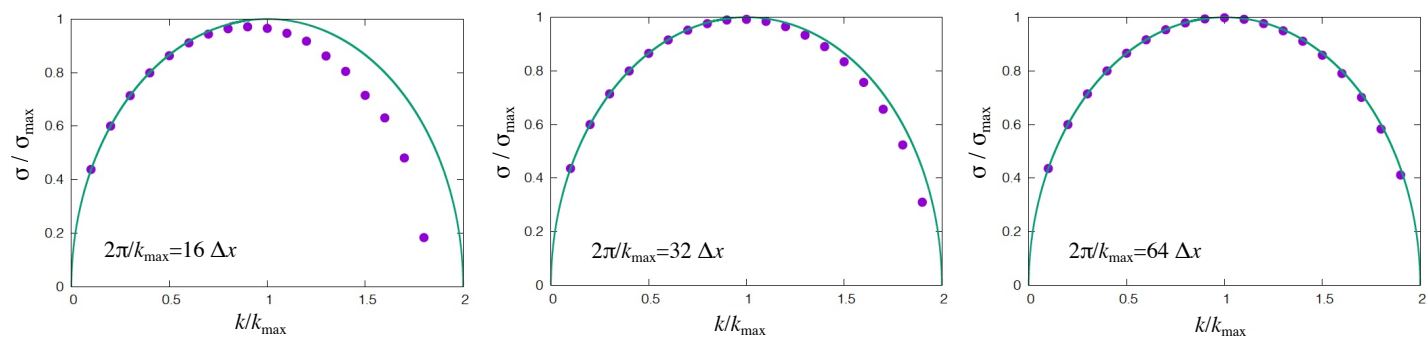

Figure 3: Result of test simulation of the Bell instability growth in linear phase (points). Solid lines are the theoretical dispersion relation under a constant CR current. From left panel to right panel, numerical spatial resolution against the most unstable scale is increased from 16 to 64 spatial cells.

We can confirm that our code can accurately capture the growth of the Bell instability, even when the most unstable scale is resolved only by 16 numerical cells.

\section{Results of Simulations}

We performed simulations of shock propagation in our CSM models, in which the most unstable scale of the Bell instability is resolved roughly 30 numerical cells. Figure 4 shows the resulting structure of the magnetic field around the shock front (left panel) and CR spectrum at shock (right panel). We see the amplified turbulent magnetic field due to the Bell instability and particle acceleration up to around $1 \mathrm{PeV}$. The spectrum of CRs is reasonable, since the DSA predicts the maximum energy of CRs as

$$
E_{\max } \sim 0.4 \times 10^{15} \mathrm{eV}\left(\frac{B}{0.3 \mathrm{G}}\right)\left(\frac{v_{\mathrm{sh}}}{10^{4} \mathrm{~km} \mathrm{~s}^{-1}}\right)^{2}\left(\frac{t}{10 \text { day }}\right) .
$$

To obtain cutoff CR energy, we fit the CR spectrum using trial function $f \propto p^{-4} \exp -\left(p c / E_{\text {cut }}\right)^{2}$, and we get $E_{\text {cut,fit }}=0.7 \mathrm{PeV}$. From top panel of Figure 4, we can read that the level of magnetic field amplification by the Bell instability is roughly factor ten that is smaller than that expected from the saturation level $\sim 100 \times B_{\text {ini. }}$. This stems from the fact that the escaping CRs that drive the Bell instability have finite spatial extent toward upstream (similar effect due to curvature of SNR shock is discussed in [8]).

In the above result, it is assumed that the CSM is created by the RSG wind of $\dot{M}=10^{-5} M_{\text {sun }}$ $\mathrm{yr}^{-1}$. However, recent observational study suggests that the mass loss rate would be two orders of magnitude larger than this value due to density enhancement of the wind prior to the explosion [9]. Because stronger CSM magnetic field is expected with the enhanced mass loss rate, we can accelerate CR more efficiently if we set the CSM with the larger mass loss rate. Figure 5 shows the same plots with Figure 4 but for a simulation with CSM model under $\dot{M}=10^{-3} M_{\text {sun }} \mathrm{yr}^{-1}$. We see that the resulting CR spectrum is more energetic than the previous model thanks to the stronger upstream magnetic field. The cutoff CR energy reaches $E_{\text {cut,fit }}=2.9 \mathrm{PeV}$ at $t=14.0$ day. 

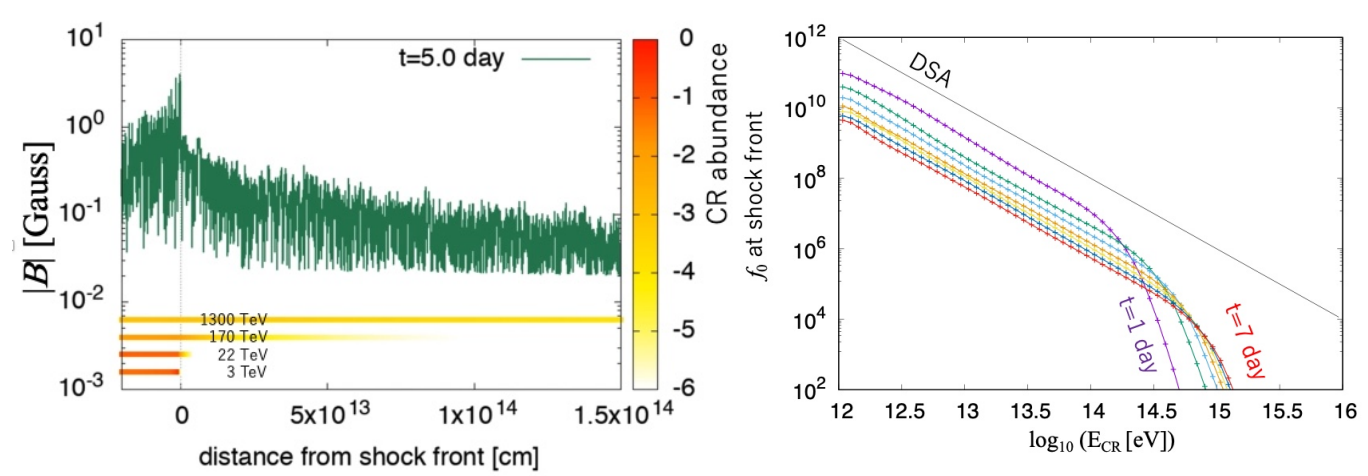

Figure 4: Structure of magnetic field around the shock front (left panel) and CR spectrum at shock (right panel) for the initial CSM model with $\dot{M}=10^{-5} M_{\text {sun }} \mathrm{yr}^{-1}$.
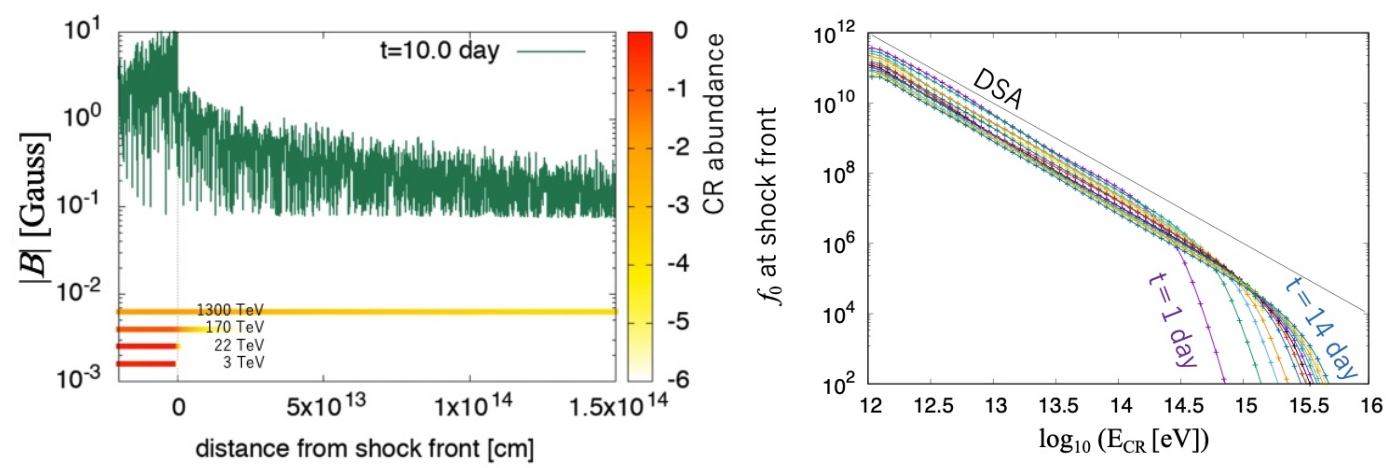

Figure 5: Structure of magnetic field around the shock front (left panel) and CR spectrum at shock (right panel) for the initial CSM model with $\dot{M}=10^{-3} M_{\text {sun }} \mathrm{yr}^{-1}$.

\section{Summary}

We have performed simulations of $\mathrm{CR}$ acceleration using a code that solves diffusion convection equation for CR and MHD equations with the effect of non-resonant CR streaming instability. We found that $\mathrm{CR}$ acceleration up to the knee energy is possible in the early supernova blast wave expansion phase in a dense CSM created by realistic RSG stellar wind. In our future works, we will take into account the effect of cosmic-ray pressure and multi dimensional effect, both of which would further enhance the maximum energy of CRs.

\section{References}

[1] Bell, A. R., Schure, K. M., Reville, B. \& Giacinti, G. 2013, MNRAS, 431, 415

[2] Inoue, T. 2019, ApJ, 872, 46

[3] Caprioli, D., \& Spitkovsky, A. 2014c, ApJ, 794, 47

[4] Roh, S., Inutsuka, S., \& Inoue, T. 2016, Astroparticle Phys., 73, 1 
[5] Marcowith, A., Dwarkadas, V. V., Renaud, M. et al. 2018, MNRAS, 479, 4470

[6] Blandford, R. D., \& Ostriker, J. P. 1978, ApJL, 221, L29

[7] Bell, A. R. 2004, MNRAS, 353, 550

[8] Schure, K. M., \& Bell, A. R. 2013, MNRAS, 435, 1174

[9] Förster, F., Moriya, T. J., Maureira, J. C. et al. 2018, Nature Astronomy, 2, 808 